\title{
Infectious mononucleosis with eyelid edema and palatal petechiae
}

\author{
Hiroaki Nakagawa ${ }^{1}$, Yasushi Miyata ${ }^{2}$, and Masato Maekawa ${ }^{1}$
}

${ }^{1}$ Division of General Medicine, ${ }^{2}$ Department of Primary Care and Community Health, Aichi Medical University, Nagakute, Japan

Received: July 8, 2020

Revised : August 25, 2020

Accepted: August 25, 2020

\section{Correspondence to}

Yasushi Miyata, M.D.

Tel: +81-561-62-3311

Fax: +81-561-62-3311

E-mail:ymymiyata@gmail.com https://orcid.org/0000-00016868-4929
A healthy 15-year-old boy presented to our clinic with a 7-day history of fever, bilateral inguinal lymphadenopathy, and night sweats. He had a mild sore throat, but no cough or rhinitis. Physical examination revealed bilateral upper eyelid edema (Fig. 1A), bilateral swelling and tenderness of the posterior cervical and inguinal lymph nodes, inflamed tonsils without a white coating, and soft palatal petechiae (Fig. 1B). He had no rash, hepatosplenomegaly, or lower leg edema. These findings suggested infectious mononucleosis (IM). Blood tests revealed 10,900/ $\mu \mathrm{L}$ leukocytes (47.0\% lymphocytes with no atypical lymphocytes), 150,000/ $\mu \mathrm{L}$ platelets, elevated liver enzymes (aspartate transaminase $118 \mathrm{IU} / \mathrm{L}$, lactate dehydrogenase 509 IU/L), Epstein-Barr virus (EBV) viral capsid antigen immunoglobulin $\mathrm{M}$ and immunoglobulin $\mathrm{G}$ antibody positive, and EBV nuclear antigen antibody negative. We therefore diagnosed IM. His symptoms resolved with symptomatic treatment, and the eyelid edema disappeared within 2 weeks (Fig. 2).

Bilateral upper eyelid edema occurs in about one-third of individuals with IM ("Hoagland sign"), typically before the onset of pharyngitis and cervical lymphadenopathy, and disappears within a few days. Palatal petechiae with IM is a useful finding (positive likelihood ratio [LR+], 5.3), but disappears in a few days. Other useful findings are posterior cervical, inguinal, or
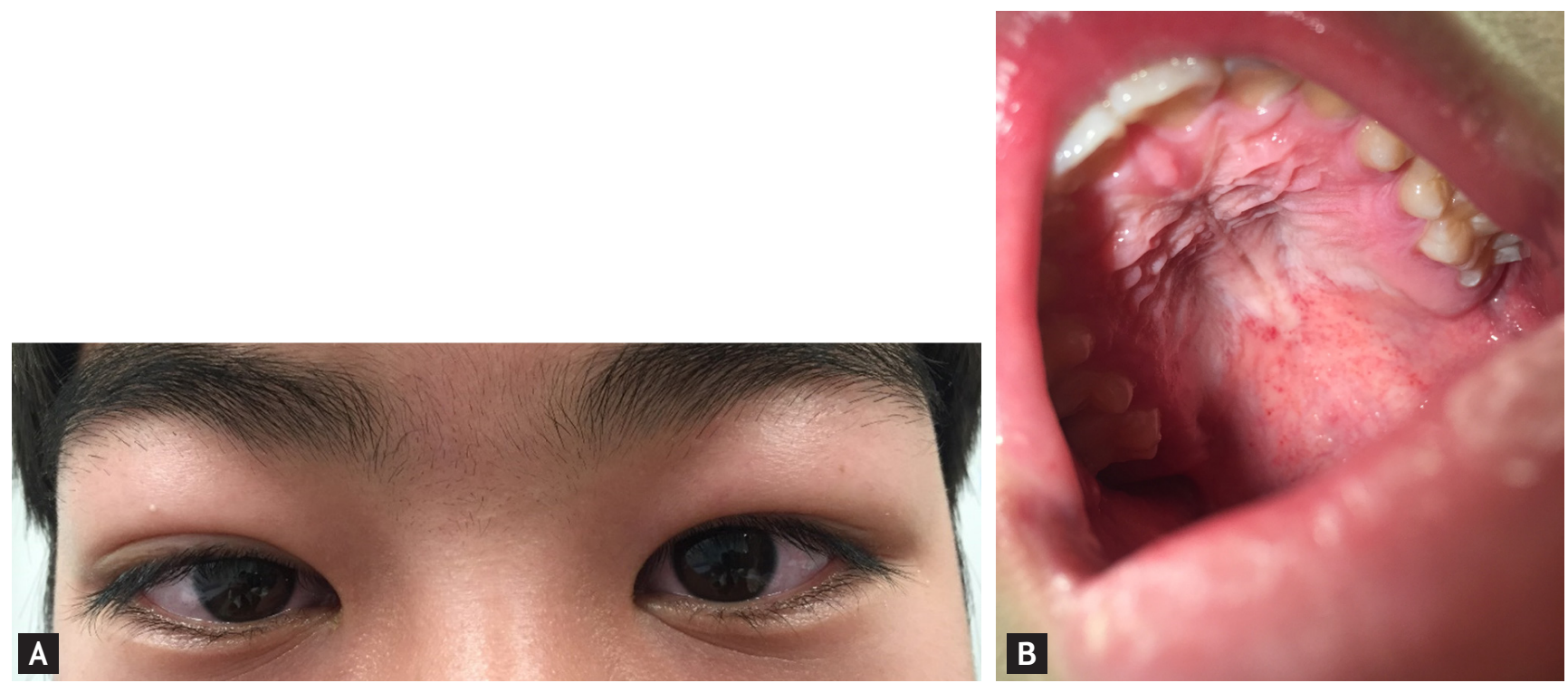

Figure 1. (A) Bilateral upper eyelid edema. (B) Soft palatal petechiae. 


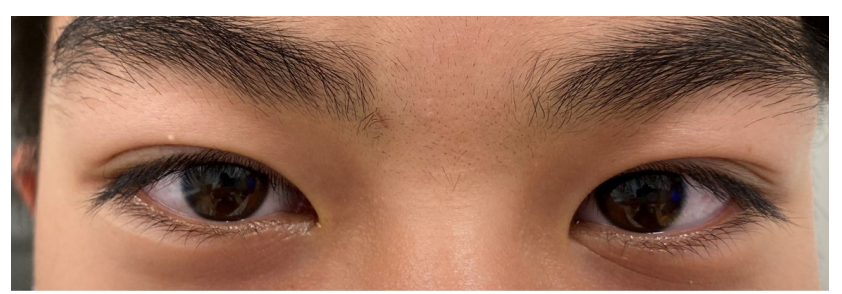

Figure 2. Eyelids after recovery.

axillary lymphadenopathy (LR+, 3.0 to 3.1), and splenomegaly (LR+, 1.9 to 6.6). Symptoms such as sore throat and malaise are of limited value for the diagnosis of IM because they are nonspecific. Night sweats are more common in individuals with IM than in those with upper respiratory infections. The combination of palatal petechiae and upper eyelid edema is useful for diagnosing IM clinically.

The patient and his family provided their consent to publish the case and the accompanying images.

\section{Conflict of interest}

No potential conflict of interest relevant to this article was reported. 